\title{
CHARACTERISTIC POLYNOMIALS OF SOME WEIGHTED GRAPH BUNDLES AND ITS APPLICATION TO LINKS
}

\author{
MOO YOUNG SOHN \\ Department of Mathematics \\ Changwon University \\ Changwon 641-773, Korea \\ and \\ JAEUN LEE \\ Department of Mathematics \\ Kyungpook National University \\ Taegu 702-701, Korea
}

(Received February 20, 1992)

\begin{abstract}
In this paper, we introduce weighted graph bundles and study their characteristic polynomial. In particular, we show that the characteristic polynomial of a weighted $K_{2}\left(\bar{K}_{2}\right)$ bundles over a weighted graph $\Gamma_{\omega}$ can be expressed as a product of characteristic polynomials two weighted graphs whose underlying graphs are $\Gamma$ As an application, we compute the signature of a link whose corresponding weighted graph is a double covering of that of a given link.
\end{abstract}

KEY WORDS AND PHRASES. Graphs, weighted graphs, graph bundles, characteristic polynomials, links, signature.

1991 AMS SUBJECT CLASSIFICATION CODES. 05C10, 05C50, 57M25.

\section{INTRODUCTION.}

Let $\Gamma$ be a simple graph with vertex set $V(\Gamma)$ and edge set $E(\Gamma)$. Let $\boldsymbol{R}$ be the field of real numbers. A weighted graph is a pair $\Gamma_{\omega}=(\Gamma, \omega)$, where $\Gamma$ is a graph and $\omega: V(\Gamma) \cup E(\Gamma) \rightarrow \boldsymbol{R}$ is a function. We call $\Gamma$ the underlying graph of $\Gamma_{\omega}$ and $\omega$ the weight function of $\Gamma_{\omega}$. In particular, if $\omega(E(\Gamma)) \subset\{1,-1\}$ and $\omega(V(\Gamma))=\{0\}$, then we call $\Gamma_{\omega}$ a signed graph.

Let $V(\Gamma)=\left\{u_{1}, \cdots, u_{n}\right\}$. The adjacency matrix of $\Gamma_{\omega}$ is an $n \times n$ matrix $A\left(\Gamma_{\omega}\right)=\left(a_{i}\right)$ defined as follows:

$$
a_{\imath \jmath}=\left\{\begin{array}{cl}
\omega(e) & \text { if } e=u_{\imath} u, \in E(\Gamma) \text { and } i \neq j, \\
\omega\left(u_{\imath}\right) & \text { if } i=j \\
0 & \text { otherwise }
\end{array}\right.
$$

for $1 \leq i, j \leq n$.

The characteristic polynomial $P\left(\Gamma_{\omega} ; \lambda\right)=\left|\lambda I-A\left(\Gamma_{\omega}\right)\right|$ of the adjacency matrix $A\left(\Gamma_{\omega}\right)$ is called the characteristic polynomial of the weighted graph $\Gamma_{\omega}$. A root of $P\left(\Gamma_{\omega} ; \lambda\right)$ is called an eigenvalue of $\Gamma_{\omega}$.

Note that if the weight function $\mathcal{L}$ of $\Gamma$ is defined by $\mathcal{L}(e)=-1$ for $e \in E(\Gamma)$ and $\mathcal{L}(u)=\operatorname{deg}(u)$ for $u \in V(\Gamma)$, where $\operatorname{deg}(u)$ denotes the degree of $u$, that is, the number of edges incident to $u$, then the weighted adjacency matrix $A\left(\Gamma_{\ell}\right)$ is called the Laplacian matrix of $\Gamma$. We call $\mathcal{L}$ the Laplacian function of $\Gamma$. The number of spanning trees of a connected graph $\Gamma$ is the 
value of any cofactor of $A\left(\Gamma_{\mathcal{L}}\right)$ [Matrux tree the orem] and in equal to the value $\frac{1}{n} \prod_{\lambda \neq 0} \lambda$, where $\lambda$ runs through all non-zero eigenvalues of $A\left(\Gamma_{\ell}\right)$ Moreores the cigenvalues of $A\left(\Gamma_{\ell}\right)$ may be used to calculate the radius of gyration of a Gaussian molecule. For more applications of the eigenvalues of $A\left(\Gamma_{\mathfrak{L}}\right)$, the reader is suggested to refer [5].

\section{WEIGHTED GRAPH BUNDLES.}

First, we introduce a weighted graph bundle. Every edge of a graph $\Gamma$ gives rise to a pain of oppositely directed edges. We denote the set of directed edges of $\Gamma$ by $D(\Gamma)$. By $\epsilon^{-1}$ we mean the reverse edge to an edge $\epsilon \in D(\Gamma)$. For any finite group $G$. a $G$-voltage assignment of $\Gamma$ is a function $\phi: D(\Gamma) \rightarrow G$ such that $\phi\left(e^{-1}\right)=\phi(c)^{-1}$ for all $f \in D(\Gamma)$. We denote the set of all $G$ voltage assignments of $\Gamma$ by $C^{1}(\Gamma ; G)$. Let $\lambda$ be another graph and let $\phi \in C^{1}(\Gamma ; A u t(\Lambda))$. where $\operatorname{Aut}(\Lambda)$ is the group of all graph automorphisms of $\Lambda$. Now, we construct a graph $\Gamma \times{ }^{\circ} \Lambda$ as follows: $\quad V\left(\Gamma \times{ }^{\phi} \Lambda\right)=V(\Gamma) \times V(\Lambda)$. Two vertices $\left(u_{1}, v_{1}\right)$ and $\left(u_{2}, v_{2}\right)$ are adjacent in $\Gamma \times{ }^{\phi} \Lambda$ if either $u_{1} u_{2} \in D(\Gamma)$ and $v_{2}=\phi\left(u_{1} u_{2}\right) v_{1}$ or $u_{1}=u_{2}$ and $v_{1} v_{2} \in E(\Lambda)$. We call $\Gamma \times{ }^{\phi} \Lambda$ the $\Lambda$-bundle over $\Gamma$ associated with $\phi$ and the natural map $p^{\phi}: \Gamma \times{ }^{\phi} \Lambda \rightarrow \Gamma$ the bundle projection. We also call $\Gamma$ and $\Lambda$ the base and the fibre of $\Gamma \times{ }^{\phi} \Lambda$, respectively. Note that the map $p^{\phi}$ maps vertices to vertices but an image of an edge can be either an edge or a vertex. If $\Lambda$ is the complement $\overline{K_{n}}$ of the complete graph $K_{n}$ of $n$ vertices, then every $\Lambda$-bundle over $\Gamma$ is an $n$-fold covering graph of $\Gamma$

Let $\Gamma_{\omega}$ and $\Lambda_{\mu}$ be two weighted graphs and let $\phi \in C^{1}(\Gamma ; A u t(\Lambda))$. We define the product of $\mu$ and $\omega$ with respect to $\phi, \omega \times{ }^{\phi} \mu$, as follows:

(1) For each vertex $(u, v)$ of $V\left(\Gamma \times{ }^{\phi} \Lambda\right),\left(\omega \times^{\phi} \mu\right)(u, v)=\omega(u)+\mu(v)$.

(2) For each edge $e=\left(u_{1}, v_{1}\right)\left(u_{2}, v_{2}\right)$ of $E\left(\Gamma \times{ }^{\phi} \Lambda\right)$,

$$
\left(\omega \times \times^{\phi} \mu\right)(e)= \begin{cases}\omega\left(u_{1} u_{2}\right) & \text { if } u_{1} u_{2} \in D(\Gamma) \text { and } v_{2}=\phi\left(u_{1} u_{2}\right) v_{1} \\ \mu\left(v_{1} v_{2}\right) & \text { if } u_{1}=u_{2} \text { and } v_{1} v_{2} \in E(\Gamma) .\end{cases}
$$

We call the weighted graph $\left(\Gamma \times{ }^{\phi} \Lambda\right)_{\omega \times} \phi_{\mu}$ the $\Lambda_{\mu}$-bundle over $\Gamma_{\omega}$ associated with $\phi$. Briefly. we call it a weighted graph bundle.

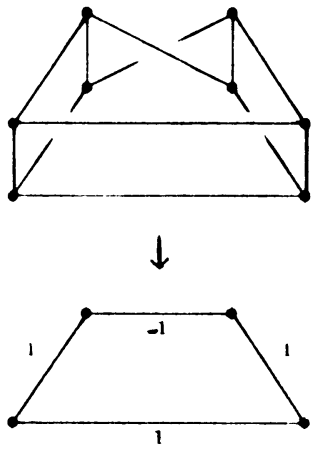

$1 x^{\phi} h_{2}$

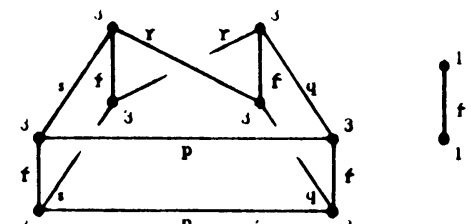

$\downarrow$

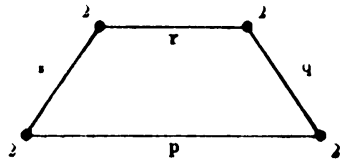

$\left(\left(c_{1} x^{\phi} h_{2}\right)_{u}+\mu\right.$

FIGURE 1. The graphs $C_{4} \times{ }^{\phi} K_{2}$ and $\left(C_{4} \times{ }^{\phi} K_{2}\right)_{\omega \times}{ }^{\phi}$.

\section{CHARACTERISTIC POLYNOMIALS}

In this section, we give a computation for the characteristic polynomial of a weighted graph bundle $\Gamma \times^{\phi} \Lambda$, where $\Lambda$ is either complete graph $K_{2}$ of two vertices or its complement $\overline{K_{2}}$, and study their related topics. Note that $A u t\left(K_{2}\right)=A u t\left(\overline{K_{2}}\right)=Z_{2}$.

For a given graph $\Gamma$ with weight function $\omega$ and for a $\phi \in C^{1}\left(\Gamma ; Z_{2}\right)$, we define a new weight function $\omega^{\phi}$ on $\Gamma$ as follows: 
(1) For $\epsilon \in E(\Gamma)$,

$$
\omega^{\phi}(\epsilon)= \begin{cases}\omega(\epsilon) & \text { if } \phi(\epsilon)=1 \\ -\omega(\epsilon) & \text { if } \phi(e)=-1\end{cases}
$$

(2) For $v \in V(\Gamma), \omega^{\phi}(v)=w(v)$.

A subgraph of $\Gamma$ is called an elementary configuration if its components are either complete graph $K_{1}$ or $K_{2}$ or a cycle $C_{m}(m \geq 3)$. We denote by $E_{k}$ the set of all elementary configurations of $\Gamma$ having $k$ vertices. In [3], the characteristic polynomial of a weighted graph $\Gamma_{\omega}$ is given as follows:

where

$$
P\left(\Gamma_{\omega} ; \lambda\right)=\sum_{k=0}^{n} a_{k}\left(\Gamma_{\omega}\right) \lambda^{n-k},
$$

$$
a_{k}\left(\Gamma_{\omega}\right)=\sum_{S \in E_{k}}(-1)^{\kappa(S)} 2^{|C(S)|} \prod_{u \in I_{v}(s)} \omega(u) \prod_{e \in I_{E}(S)} \omega(e)^{2} \prod_{e \in C(S)} \omega(e) .
$$

In the above equation, symbols have the following meaning: $\kappa(S)$ is the number of components of $S, C(S)$ the set of all cycles, $C_{m}(m \geq 3)$, in $S$, and $I_{v}(S)\left(I_{E}(S)\right)$ is the set of all isolated vertices (edges) in $S$. Moreover, the product over empty index set is defined to be 1 .

For a fixed voltage assignment $\phi \in C^{1}\left(\Gamma ; Z_{2}\right)$, we denote by $E_{\phi-1}$ the set of edges of $\Gamma$ such that $\phi(e)=-1$, i.e., $E_{\phi-1}=\{e \in E(\Gamma): \phi(e)=-1\}$. Let $\Gamma\left(E_{\phi-1}\right)$ be the edge subgraph of $\Gamma$ induced by $E_{\phi-1}$ having weight zero in vertices. If $\Gamma_{\omega}$ is a weighted graph, then the weight function of its subgraph $S$ is the restriction of $\omega$ on $S$.

THEOREM 1. Let $\overline{K_{2}}$ be a constant weighted graph, say $\mu(v)=c$ for $v \in \bar{K}_{2}$. Then, for each $\phi \in C^{1}\left(\Gamma ; Z_{2}\right)$, we have

$$
P\left(\left(\Gamma \times{ }^{\phi} \overline{K_{2}}\right)_{\omega \times \phi_{c}} ; \lambda\right)=P\left(\Gamma_{\omega} ; \lambda-c\right) P\left(\Gamma_{\omega^{\phi}} ; \lambda-c\right) .
$$

PROOF. Let $A\left(\Gamma_{\omega}\right)$ be the adjacency matrix of $\Gamma_{\omega}$ and let $A\left(\Gamma_{\omega^{\phi}}\right)$ the adjacency matrix of $\Gamma_{\omega^{\phi}}$. Then we have

$$
\begin{aligned}
& A\left(\Gamma_{\omega}\right)=A\left(\left(\Gamma \backslash\left(E_{\phi-1}\right)\right)_{\omega}\right)+A\left(\Gamma\left(E_{\phi-1}\right)_{\omega}\right), \\
& A\left(\Gamma_{\omega^{\phi}}\right)=A\left(\left(\Gamma \backslash\left(E_{\phi-1}\right)\right)_{\omega}\right)-A\left(\Gamma\left(E_{\phi-1}\right)_{\omega}\right) .
\end{aligned}
$$

Let $V\left(\Gamma \times{ }^{\phi} \bar{K}_{2}\right)=\left\{\left(u_{1}, 1\right), \cdots,\left(u_{n}, 1\right),\left(u_{1},-1\right), \cdots,\left(u_{n},-1\right)\right\}$. If is not difficult to show that

$$
\begin{aligned}
A\left(\left(\Gamma \times{ }^{\phi} \overline{K_{2}}\right)_{\omega \times} \phi_{c}\right)= & \left(A\left(\Gamma_{\omega}\right)-A\left(\Gamma\left(E_{\phi-1}\right)_{\omega}\right)+\left[\begin{array}{lll}
c & & 0 \\
& c & \\
& & \ddots \\
0 & & c
\end{array}\right]\right) \otimes\left[\begin{array}{ll}
1 & 0 \\
0 & 1
\end{array}\right] \\
& +\left(A\left(\Gamma\left(E_{\phi-1}\right)_{\omega}\right)\right) \otimes\left[\begin{array}{ll}
0 & 1 \\
1 & 0
\end{array}\right]
\end{aligned}
$$

Let $M$ be a regular matrix of order 2 satisfying

$$
M^{-1}\left[\begin{array}{ll}
0 & 1 \\
1 & 0
\end{array}\right] M=\left[\begin{array}{cc}
1 & 0 \\
0 & -1
\end{array}\right]
$$

Put

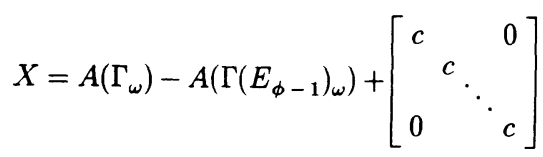

$$
\begin{aligned}
& Y=A\left(\Gamma\left(E_{\phi-1}\right)_{\omega}\right) .
\end{aligned}
$$


Then

$$
\begin{aligned}
& \left(I c M^{-1}\right) A\left(\left(\Gamma \times{ }^{\phi} \overline{K_{2}}\right)_{\omega \times \phi_{c}}\right)(I: M) \\
& =\left[\begin{array}{cc}
X+Y & 0 \\
0 & X-Y
\end{array}\right]
\end{aligned}
$$

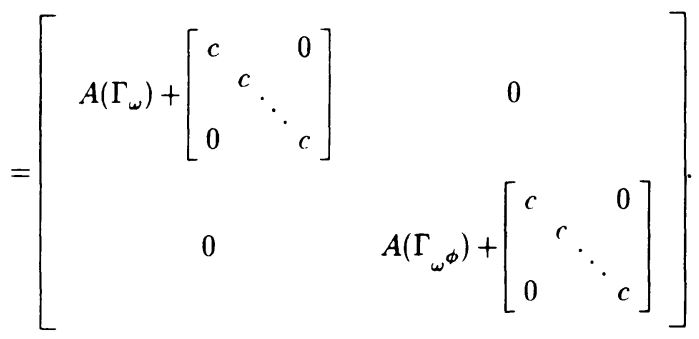

Since $\left|\left(I \otimes M^{-1}\right)(I \otimes M)\right|=1$ and

$$
\left|\left[\lambda I-A\left(\left(\Gamma \times \bar{K}^{\phi}\right)_{\omega \times} \phi_{c}\right)\right]\right|=\left|\left[\lambda I-\left(I \otimes M^{-1}\right) A\left(\left(\Gamma \times{ }^{\phi} \overline{K_{2}}\right)_{\omega \times} \phi_{c}\right)(I \otimes M)\right]\right|
$$

we have our theorem.

THEOREM 2. Let $K_{2_{\mu}}=\left(K_{2}, \mu\right)$ be a weighted graph having constant weight on vertices. Then, for each $\phi \in C\left(\Gamma ; Z_{2}\right)$, we have

$$
P\left(\left(\Gamma \times{ }^{\phi} K_{2}\right)_{\omega \times{ }^{\phi}{ }_{\mu}} ; \lambda\right)=P\left(\Gamma_{\omega} ; \lambda-c_{v}-c_{e}\right) P\left(\Gamma_{\omega^{\phi}} ; \lambda-c_{v}+c_{e}\right),
$$

where $c_{v}=\mu\left(v_{1}\right)=\mu\left(v_{2}\right)$ for the vertices $v_{1}, v_{2}$ and $c_{e}=\mu(e)$ for the edge $e$ in $K_{2}$.

PROOF. Clearly, we have

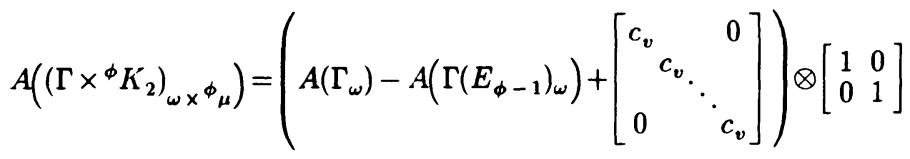

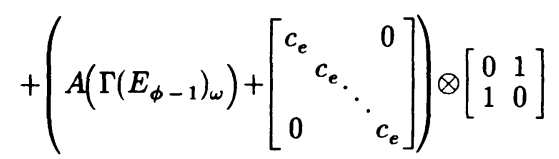

where $c_{v}=\mu\left(v_{1}\right)=\mu\left(v_{2}\right)$ and $c_{e}=\mu(e)$ for the edge $e$ in $K_{2}$. Let $M$ be a regular matrix of order 2 satisfying

Then

$$
M^{-1}\left[\begin{array}{ll}
0 & 1 \\
1 & 0
\end{array}\right] M=\left[\begin{array}{cc}
1 & 0 \\
0 & -1
\end{array}\right]
$$

$$
\begin{aligned}
& \left(I \otimes M^{-1}\right) A\left(\left(\Gamma \times{ }^{\phi} K_{2}\right)_{\omega \times} \phi_{\mu}\right)(I \otimes M)
\end{aligned}
$$

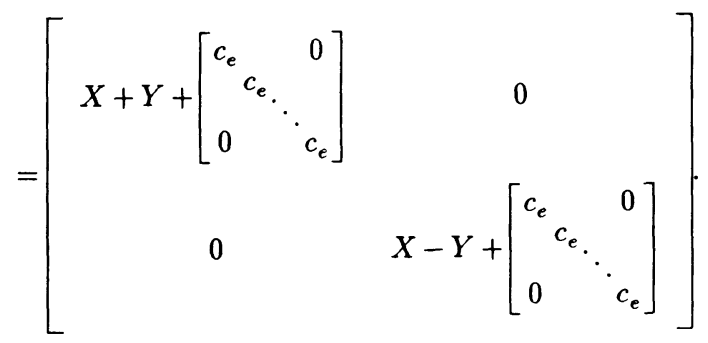




$$
=\left[\begin{array}{cc}
A\left(\Gamma_{\omega}\right)+Z_{1} & 0 \\
0 & A\left(\Gamma_{\omega^{\phi}}\right)+Z_{2}
\end{array}\right]
$$

where $X$ and $Y$ are the same matrices as in the proof of Theorem 1 and for $\imath=1,2$,

$$
Z_{1}=\left[\begin{array}{ccc}
c_{v}+(-1)^{2-1} c_{e} & 0 \\
& c_{v}+(-1)^{t-1} c_{e} & \\
& & c_{v}+(-1)^{2-1} c_{e}
\end{array}\right]
$$

Using method similar to the proof of Theorem 1, we have our theorem.

Note that for any $\phi \in C^{1}(\Gamma ; A u t(\Lambda))$, the Laplacian function of $\Gamma \times{ }^{\phi} \Lambda$ is the product of Laplacian functions of $\Gamma$ and $\Lambda$ with respect to $\phi$. Clearly, the Laplacian function of the $\overline{K_{2}}$ is the zero function; and the Laplacian function of the $K_{2}$ has value 1 and -1 for each of its vertices and its edge, respectively. We shall denote the Laplacian function of a graph by $\mathcal{L}$ if it makes no confusion. Then Theorem 1 and Theorem 2 give the following corollary.

COROLLARY 1. For any $\phi \in C^{1}\left(\Gamma ; Z_{2}\right)$,

(1) $P\left(\left(\Gamma \times{ }^{\phi} \overline{K_{2}}\right)_{\ell} ; \lambda\right)=P\left(\Gamma_{\ell} ; \lambda\right) P\left(\Gamma_{\ell^{\phi}} ; \lambda\right)$.

(2) $\quad P\left(\left(\Gamma \times{ }^{\phi} K_{2}\right)_{\ell} ; \lambda\right)=P\left(\Gamma_{\ell} ; \lambda\right) P\left(\Gamma_{\ell^{\phi}} ; \lambda-2\right)$.

Now, we consider another invariant of weighted graphs called the signature. Since $A\left(\Gamma_{\omega}\right)$ is symmetric, $A\left(\Gamma_{\omega}\right)$ can be diagonalized through congruence over $\boldsymbol{R}$. Let $d_{+}$denote the number of positive diagonal entries, and $d_{-}$the number of negative diagonal entries. The signature of a weighted graph $\left(\Gamma_{\omega}\right)$ is defined by $\sigma\left(A\left(\Gamma_{\omega}\right)\right)=d_{+}-d_{-}$and is denoted by $\sigma\left(\Gamma_{\omega}\right)$. It is an invariant for weighted 2-isomorphic graphs (see [7]).

From now on, we will consider the weight function on $\overline{K_{2}}$ as zero function and the weight function $\mu$ on $K_{2}$ as the map defined by $\mu(v)=0$ for each $v \in V\left(K_{2}\right)$ and $\mu(e)=c_{e}$ for the edge $e$ of $K_{2}$. Then we can compute the signature of a double covering of $\Gamma$.

COROLLARY 2. $\sigma\left(\left(\Gamma \times{ }^{\phi} \overline{K_{2}}\right)_{\omega \times} \phi_{0}\right)=\sigma\left(\Gamma_{\omega}\right)+\sigma\left(\Gamma_{\omega^{\phi}}\right)$ for $\phi \in C^{1}\left(\Gamma ; Z_{2}\right)$.

For convenience, we adapt the following notations. For a real number $c$, a weighted graph $\Gamma_{\eta}$ and an eigenvalue $\lambda$ of $\Gamma_{\eta}$,

$$
\begin{gathered}
P(c)_{\eta}^{-}=\{\lambda<0: \lambda+c>0\}, \\
P(c)_{\eta}^{+}=\{\lambda>0: \lambda+c>0\}, \\
Z(c)_{\eta}=\{\lambda \neq 0: \lambda+c=0\}, \\
N(c)_{\eta}^{-}=\{\lambda<0: \lambda+c<0\}, \\
N(c)_{\eta}^{+}=\{\lambda>0: \lambda+c<0\} .
\end{gathered}
$$

We also denote the multiplicity of $\lambda$ by $m_{\eta}(\lambda)$.

By using the above notations and Theorem 2, we get the signature of a $K_{2}$-bundle over $\Gamma$.

COROLLARY 3. For $\phi \in C^{1}\left(\Gamma ; Z_{2}\right)$,

(1) if $c_{e} \geq 0$, then

$$
\begin{aligned}
& \sigma\left(\left(\Gamma \times{ }^{\phi} K_{2}\right)_{\omega \times} \phi_{\mu}\right)=\sigma\left(\Gamma_{\omega}\right)+\sigma\left(\Gamma_{\omega^{\phi}}\right)
\end{aligned}
$$

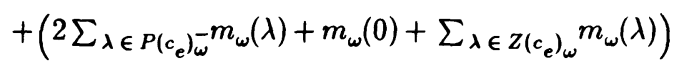

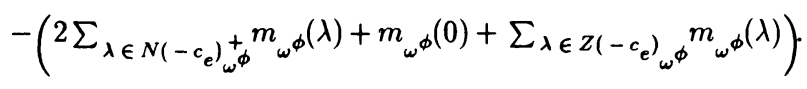


(2) if $c_{e}<0$, then

$$
\begin{aligned}
& \sigma\left(\left(\Gamma \times{ }^{\phi} K_{2}\right)_{\omega \times{ }^{\phi}}\right)=\sigma\left(\Gamma_{\omega}\right)+\sigma\left(\Gamma_{\omega^{c}}\right) \\
& -\left(2 \sum_{\lambda \in N^{\prime}\left(c_{e}\right)_{\omega}^{+}} m_{\omega}(\lambda)+m_{\omega}(0)+\sum_{\lambda \in Z\left(c_{e}\right)_{\omega}} m_{\omega}(\lambda)\right)
\end{aligned}
$$

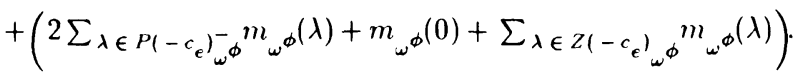

REMARK. Though the results in this section stated only for a simple graph, it remains true for any graph.

\section{APPLICATIONS TO LINKS.}

In a signed graph $\Gamma_{\omega}$, an edge $e$ of $\Gamma$ is said to be positive if $\omega(e)=1$ and negative otherwise. For a signed graph $\Gamma_{\omega}$, we define a new weight function $\widetilde{\omega}$ of $\Gamma$ by $\widetilde{\omega}(e)=\omega(e)$ for any edge $e \in \Gamma_{\omega}$ and $\widetilde{\omega}\left(u_{\imath}\right)=\sum_{j=1, i \neq j}^{n} a_{\imath}$, where $a_{\imath}$ is the number of positive edges minus the number of negative edges which have two end vertices $u_{\imath}$ and $u_{j}$. Given a knot or link $L$ in $R^{3}$, we project it into $R^{2}$ so that each crossing point has proper double crossing. The image of $L$ is called a link (or knot) diagram of $L$, and we do not distinguish between a diagram and the image of $L$.

We may assume without loss of generality that a link diagram $\widetilde{L}$ of $L$ intersects itself transversely and has only finitely many crossings. The link diagram $\widetilde{L}$ divides $\boldsymbol{R}^{2}$ into finitely many domains, which will be classified as shaded or unshaded. No two shaded or unshaded domains have an edge in common. We now construct a signed planar graph $\Gamma_{\omega}$ from $\widetilde{L}$ as follows: take a point $v_{\mathfrak{t}}$ from each unshaded domain $D_{\mathfrak{v}}$. These points form the set of vertices $V\left(\Gamma_{\omega}\right)$ of $\Gamma_{\omega}$. If the boundaries of $D_{\mathbf{\imath}}$ and $D$, intersect $k$-times, say, crossing at $c_{\ell_{1}}, c_{\ell_{2}}, \cdots, c_{\ell_{k}}$, then we form multiple edges $e_{\ell_{1}}, e_{\ell_{2}}, \cdots, e_{\ell_{k}}$ on $\boldsymbol{R}^{2}$ with common end vertices $v_{\imath}$ and $v_{\jmath}$, where each edge $e_{\ell_{m}}$ passes through a crossing $c_{\ell_{m}}$, for $m=1,2, \cdots, k$. To define the weight of an edge, first, we define the index $\epsilon(c)$ to each crossing $c$ of the link diagram as in Figure 2. To each edge of $\Gamma$ passes through exactly one crossing, say $c$, of $\widetilde{L}$, the weight $\omega(e)$ will be defined as $\omega(e)=\epsilon(c)$. (See Figure 3).

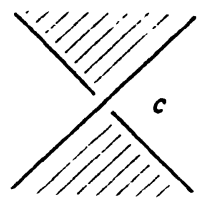

$f(c) \cdots+1$

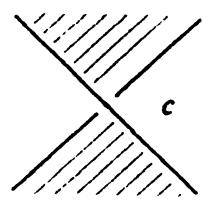

$f(c)=-1$

FIGURE 2. The index $\epsilon(c)$. 

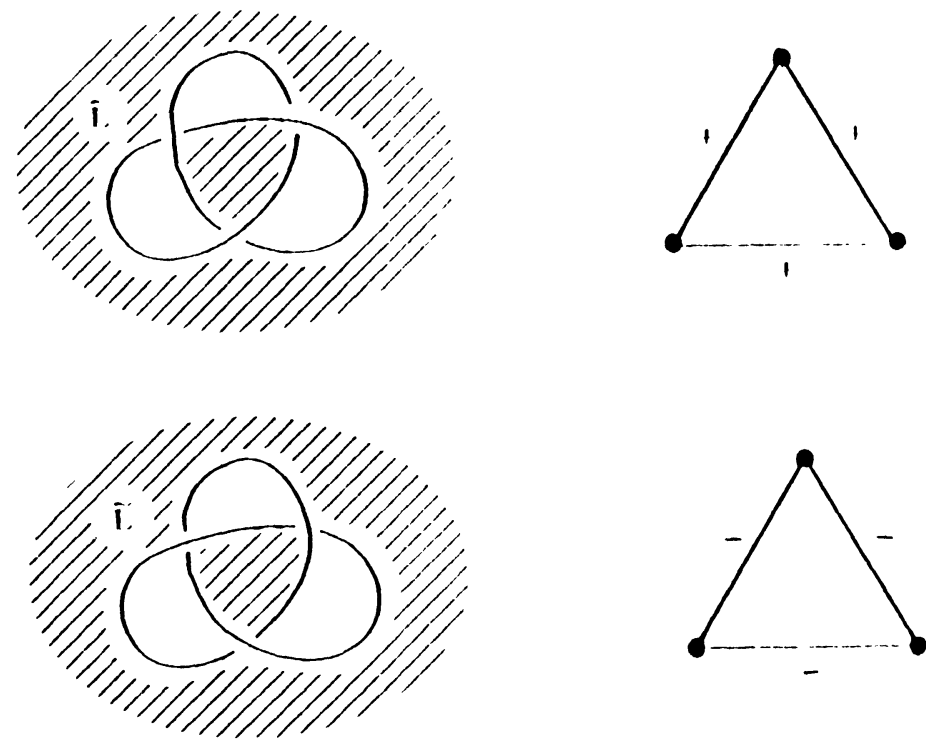

FIGURE 3. The correspondence between $\widetilde{L}$ and $\Gamma_{\omega}(\widetilde{L})$.

The resulting signed planar is called the graph of a link with respect to $\widetilde{L}$ and is denoted by $\Gamma_{\omega}(\widetilde{L})$. The signed planar graph $\Gamma_{\omega}(\widetilde{L})$ depends not only on $\widetilde{L}$ but also on shading. Conversely, given a signed planar graph $\Gamma_{\vartheta}$, one can construct uniquely the link diagram $L\left(\widetilde{L}{ }_{\vartheta}\right)$ of a link so that $\Gamma_{\omega}\left(L\left(\widetilde{\Gamma}_{\vartheta}\right)\right)=\Gamma_{\vartheta}$.

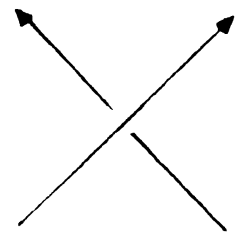

$\omega(c)=1$

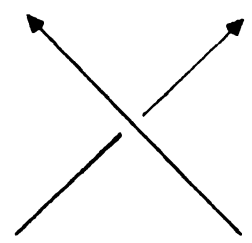

$\omega(c)=-1$

FIGURE 4. The index $\omega(c)$.

Suppose that we are given an oriented link $L$. The orientation of $L$ induces the orientation of a diagram $\widetilde{L}$. We then define the second index $\omega(c)$, called the twist or writhe at each crossing $c$ as show in Figure 4. We now need the third index $\eta_{\rho}(c)$ at crossing $c$. Let $\widetilde{L}$ be an oriented diagram and $\rho$ shading on $\widetilde{L}$. Let $\eta_{\rho}(c)=\omega(c) \delta_{e(c) \omega(c)}$, where $\delta$ denotes Kronecker's delta. We define $\eta_{\rho}(\widetilde{L})=\sum \eta_{\rho}(c)$, where the summation runs over all crossing in $\widetilde{L}$. The index $\eta_{\rho}(\widetilde{L})$ depends not only on the shading $\rho$ but also on the orientation of $\widetilde{L}$. The following Lemma can be found in ([7], [4]).

LEMMA 1. The signature $\sigma(L)$ of a link $L$ is $\sigma(L)=\sigma(\Gamma(\widetilde{L}))-\eta_{\rho}(\widetilde{L})$.

Let $\widetilde{L}_{1}$ and $\widetilde{L}_{2}$ be link diagrams of $L_{1}$ and $L_{2}$, respectively. The link $L_{2}$ is called a double covering of the link $L_{1}$ if $\Gamma_{\omega}\left(\widetilde{L}_{2}\right)$ is a double covering of $\Gamma_{\omega}\left(\widetilde{L}_{1}\right)$ as weighted graphs and it can be extended to a branched covering on $R^{2}$. Let $\phi$ be a voltage assignment in $C^{1}\left(\Gamma_{\omega}(\widetilde{L}) ; Z_{2}\right)$ such that $\phi(e)=-1$ for some edge $e$ and $\phi(e)=1$ otherwise, then $\Gamma_{\omega}(\widetilde{L}) \times{ }^{\phi} \overline{K_{2}}$ is a planar double covering of $\Gamma_{\omega}(\widetilde{L})$ of which the corresponding link is a double covering of $L$.

Therefore, one can construct the double covering link diagram $\widetilde{L}\left(\Gamma_{\omega}(\tilde{\widetilde{L}}) \times{ }^{\phi} \overline{K_{2}}\right)$ of $\widetilde{L}$. Moreover, we can give an orientation on $\widetilde{L}\left(\Gamma_{\omega}(\widetilde{L}) \times{ }^{\phi} \overline{K_{2}}\right)$ so that the covering map from $\widetilde{L}$ to $\widetilde{L}\left(\Gamma_{\omega}(\widetilde{L}) \times{ }^{\phi} \overline{K_{2}}\right)$ preserves the orientation. We have $\eta_{\rho}\left(\widetilde{L}\left(\Gamma_{\omega}(\widetilde{L}) \times{ }^{\phi} \overline{K_{2}}\right)\right)=2 \eta_{\rho}(\widetilde{L})$ (see Figure $5)$. 


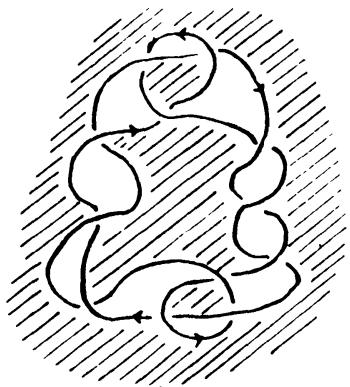

$\eta\left(L\left(\Gamma_{0}(\tilde{L}) \times+K_{2}\right)\right)=-4$

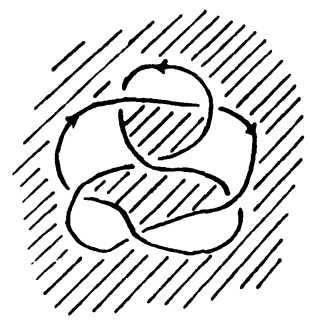

$\eta(\bar{L})=-2$

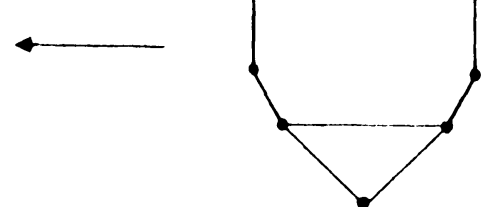

$r_{1}\left(i_{i}\right) \times \pi_{2}$
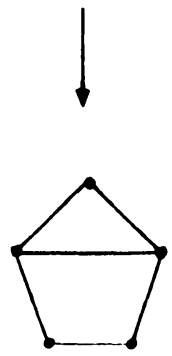

$\Gamma_{*}(i)$

FIGURE 5. Covering graph and covering link.

Therefore, by using Lemma 1 and Corollary 2, we get the following theorem.

THEOREM 3. For any oriented link diagram $\widetilde{L}$,

$$
\sigma\left(\widetilde{L}\left(\Gamma_{\omega}(\widetilde{L}) \times{ }^{\phi} \overline{K_{2}}\right)\right)=\sigma\left(\Gamma_{\omega}(\widetilde{L})\right)+\sigma\left(\Gamma_{\omega^{\phi}}(\widetilde{L})\right)-2 \eta_{\rho}(\widetilde{L})
$$

for each $\phi \in C^{1}\left(\Gamma ; Z_{2}\right)$ such that $\phi(e)=-1$ for some edge $e \in \Gamma_{\omega}(\widetilde{L})$ and $\phi(e)=1$ otherwise.

ACKNOWLEDGEMENT. The first author was supported by KOSEF and the second author was supported by TGRC-KOSEF.

\section{REFERENCES}

1. BIGGS, N., Algebraic Graph Theory, Cambridge University Press, 1974.

2. CHAE, Y.; KWAK, J.H. and LEE, J., Characteristic polynomials of some graph bundles, preprint.

3. CVETKOVIĆ, D.M.; DOOB, M. and SACHS, H., Spectra of Graphs, Academic Press, New York, 1979.

4. GORDON, C.M. and LITTERLAND, R.A., On the signature of a link, Invent. Math. 47 (1978), 53-69.

5. GRONE, R.; MERRIS, R. and SUNDER, V.S., The Laplacian spectrum of a graph, SIAM J. Matrix Anal. Appl. 11 (1990), 218-238.

6. KWAK, J.H. and LEE, J., Isomorphism classes of graph bundles, Canad. J. Math. 42 (1990), 747-761.

7. MURASUGI, K., On invariants of graphs with applications to knot theory, Trans. Amer. Math. Soc. 314 (1989), 1-49.

8. MURASUGI, K., On the signature of graphs, C.R. Math. Rep. Acad. Sci. Canada, 10 (1989), 107-111.

9. MURASUGI, K., On the certain numerical invariant of link type, Trans. Amer. Math. Soc. 117 (1965), 387-422.

10. SCHWENK, A.J., Computing the characteristic polynomial of a graph, Lecture Notes in Mathematics 406, Springer-Verlag (1974), 153-172. 


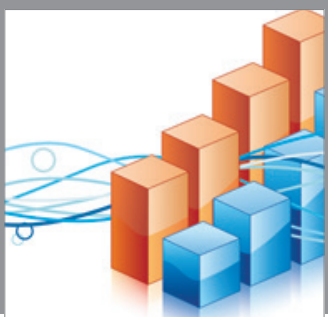

Advances in

Operations Research

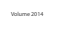

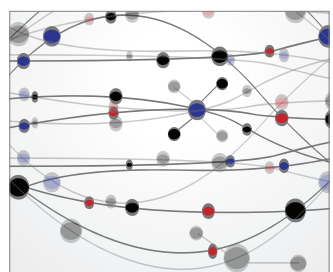

\section{The Scientific} World Journal
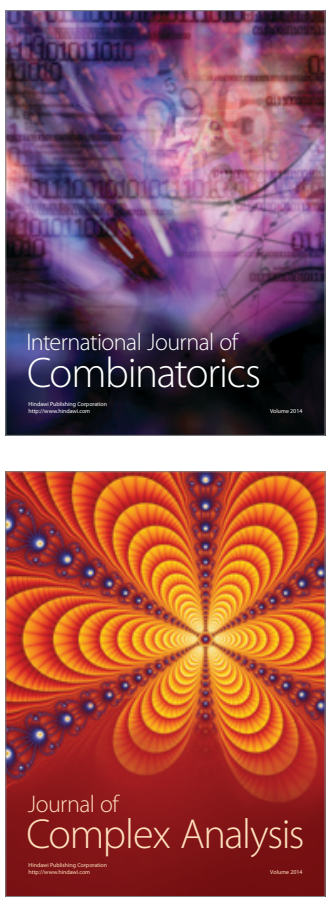

International Journal of

Mathematics and

Mathematical

Sciences
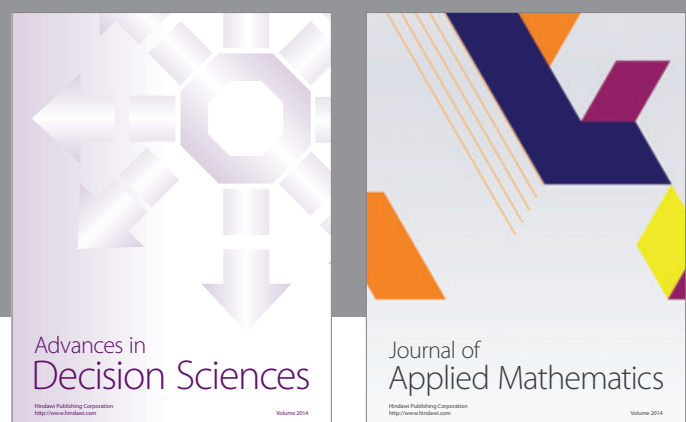

Journal of

Applied Mathematics
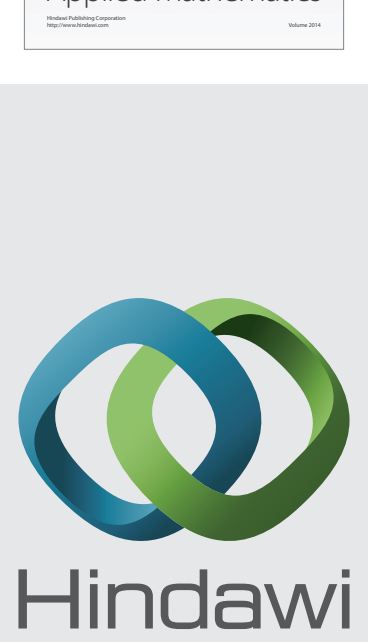

Submit your manuscripts at http://www.hindawi.com
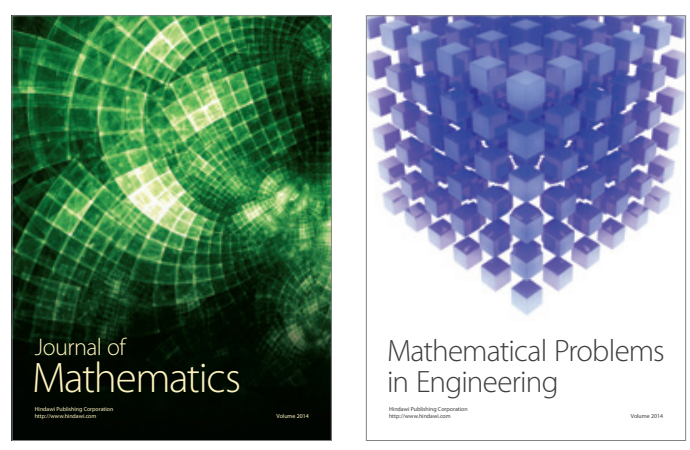

Mathematical Problems in Engineering
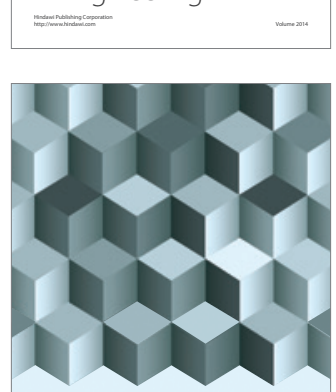

Journal of

Function Spaces
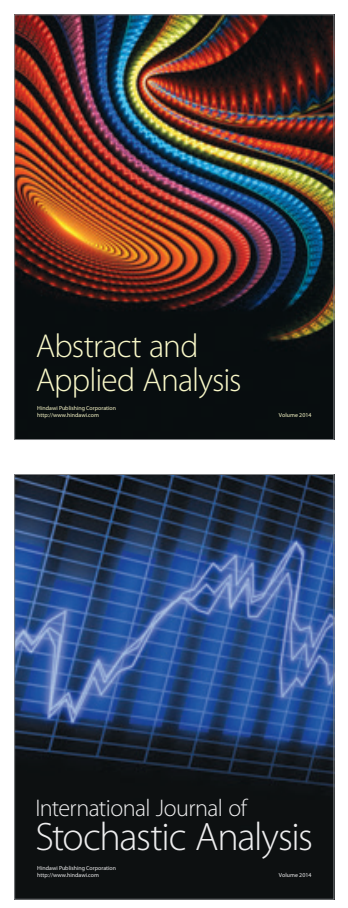

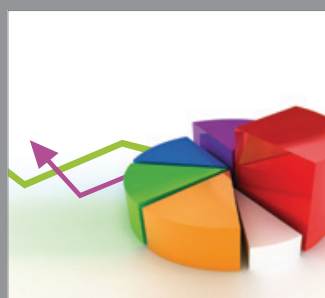

ournal of

Probability and Statistics

Promensencen
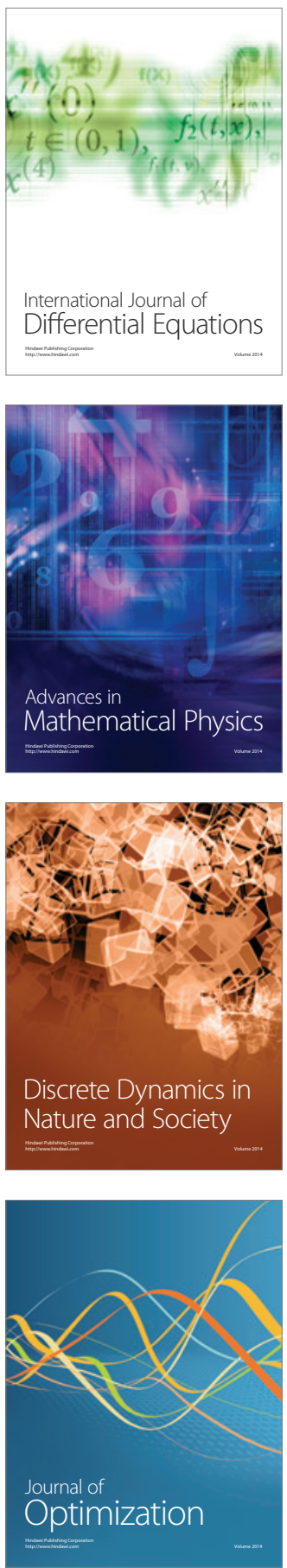